\title{
Local Extensions in Singular Space-Times
}

\author{
C. J. S. Clarke \\ Department of Applied Mathematics and Theoretical Physics. Cambridge, U.K.
}

Received February 12, 1973

\begin{abstract}
A space-time has a local extension through a point on its b-boundary if and only if an appropriate number of covariant derivatives of the Riemann tensor have limiting values on a curve ending at the boundary-point, measured in a parallely propagated tetrad. The extension has the same differentiability as the space-time if the curve is "reasonable" in a well-defined sense.
\end{abstract}

\section{Introduction}

In General Relativity a space-time $(M, g)$ is usually called singular [1] when it is incomplete; the most general sense of this being that it possesses curves $\kappa$ inextensible at one end with finite length when measured with a parallely propagated tetrad $\{\underset{i}{X}\}$ (i.e. $\left.\int\left[\sum_{i}(g(\underset{i}{X}, \dot{\kappa}))^{2}\right]^{1 / 2} \mathrm{~d} s<\infty\right)$.

By Schmidt's [2] construction, such a curve defines a point on a boundary that can be attached to $M$, the b-boundary. Points on the b-boundary can arrise merely from "cutting out" part of a space-time: such points disappear when the removed part is replaced, which leads us to classify boundary points into those which can be so disposed of by an extension, and those for which this is impossible. Only a point of the latter type should be regarded as a true singularity.

Next one seeks to characterise those boundary-points where an extension is possible. In a forthcoming paper [3] Ellis and Schmidt split the problem into two questions. First, is a local extension (to be defined shortly) possible? Secondly, do the local extensions give rise to a true extension? In the present paper we give sufficient conditions for the existence of local extensions of differentiability class $C^{2}$ and $C^{k}$, where $k$ $(3 \leqq k \leqq \infty)$ is the differentiability of the metric on $M$. It is shown that, provided the boundary-point is accessible by a curve which is not too pathological (e.g. a causal curve), then a local $C^{k}$ extension is possible if and only if the components of all relevant covariant derivatives of the

* Work supported by a fellowship under the European Programme of the Royal Society at the I. Institut für theoretische Physik. University of Hamburg. 
Riemann tensor, in a parallely propagated tetrad, tend to limits on the curve. An example will be given to show that in general this only ensures a $C^{2}$ extension.

\section{Terminology and Notation}

For simplicity we shall phrase the results in terms of a general (pseudo-) Riemannian metric. Thus $\mathscr{G}^{k}(M)$ will denote the set of all $C^{k}$ metrics of a given signature on a paracompact Hausdorff manifold $M$ of dimension $n, n \geqq 3$, with $k$ possibly $\infty$.

Let $(M, g),\left(M^{\prime}, g^{\prime}\right)$ be two (pseudo-) Riemannian spaces, $L$ and $L^{\prime}$, respectively, their (pseudo-) orthonormal frame bundles and $\theta^{i}$ and $\theta^{i \prime}$ the standard horizontal forms on these bundles: if $X \in T_{z}(L)$ with $z=\left\{\begin{array}{l}X \\ 1\end{array}, \ldots, \underset{n}{X}\right\}$ then we can write $\pi_{*} X=\xi^{i} \underset{i}{X}$ and $\theta^{i}(X):=\xi^{i}$. Indices $i, j, \ldots$ run from 1 to $n$. Immersions $\gamma, \gamma^{\prime}$ of $I:=(0,1)$ in $L, L^{\prime}$ will be called equivalent if, for all $s, \theta^{i}(\dot{\gamma}(s))=\theta^{i}\left(\dot{\gamma}^{\prime}(s)\right)$. Immersions $\kappa, \kappa^{\prime}$ of $I$ into $M, M^{\prime}$ will be called equivalent (relative to $\gamma, \gamma^{\prime}$ ) if $\gamma$ and $\gamma^{\prime}$ are equivalent horizontal lifts of $\kappa, \kappa^{\prime}$ respectively.

Let $\kappa, \kappa^{\prime}$ be equivalent proper embeddings relative to horizontal lifts $\gamma, \gamma^{\prime}$. Then we define a map $\tau_{\gamma \gamma^{\prime}}: U_{\gamma \gamma^{\prime}} \rightarrow U_{\gamma \gamma^{\prime}}^{\prime}$, where $U_{\gamma \gamma^{\prime}}$ and $U_{\gamma \gamma^{\prime}}^{\prime}$ are neighbourhoods of $\kappa(I)$ and $\kappa^{\prime}(I)$, respectively, as follows. The bundle metric [2] defines a field of orthogonal surface elements along $\gamma$. The projection of this yields a field of transverse surface elements on $\kappa(I)$. There is a neighbourhood $\tilde{U}$ of $\kappa(I)$ such that for every point $x$ in $\tilde{U}$ there is a unique point $s_{x} \in I$ and a unique geodesic joining $x$ and $\kappa\left(s_{x}\right)$, whose tangent vector $Z_{x}$ at $\kappa\left(s_{x}\right)$ lies in one of these transverse surface elements. Choose $Z_{x}$ so that $\exp Z_{x}=x$. Define $\tilde{U}^{\prime}$ similarly in $M^{\prime}$ with respect to $\kappa^{\prime}$.

For $Z \in T_{\kappa(s)}(M)$ let $\dot{Z}$ be the horizontal lift of $Z$ at $\gamma(s)$ and let $\tilde{Z}^{\prime}$ be the horizontal vector at $\gamma^{\prime}(s)$ for which $\theta^{i}(\tilde{Z})=\theta^{i}\left(\tilde{Z}^{\prime}\right)$. Set $t(Z):=\pi_{*}\left(\tilde{Z}^{\prime}\right)$ where $\pi$ is the projection in $L$. Finally we define $U_{\gamma \gamma^{\prime}}$ as the subset of points $x$ in $\tilde{U}$ such that $\tau(x) \in \tilde{U}^{\prime}$, where $\tau(x):=\exp t\left(Z_{x}\right)$, and set $U_{\gamma \gamma^{\prime}}^{\prime}:=\tau\left(U_{\gamma \gamma^{\prime}}\right)$.

To specify the transverse surface element field explicitly, choose vectors $\underset{\alpha}{V}(\alpha=1,2, \ldots, n-1)$ at a point $x_{0}=\kappa\left(s_{0}\right)$ satisfying

where

$$
\sum_{i} V_{\alpha}^{i} V^{i}=\delta_{\alpha \beta}, \quad \sum_{i} V_{x}^{i} Y^{i}=0
$$

$$
\begin{aligned}
& \gamma(s)=:\{\underset{1}{X}(s), \ldots, \underset{n}{X}(s)\}, \quad Y^{i} \equiv Y^{i}(s):=\dot{\kappa}(s)^{i} \\
& =\theta^{i}(\dot{\gamma}(s))
\end{aligned}
$$


and for any vector $A, A^{i}$ are the components relative to the $\underset{i}{X}$ :

$$
A=A_{i}^{i} X_{i}
$$

Then (1) is preserved by the propagation equations

$$
\frac{\mathrm{d} V_{\alpha}^{i}}{\mathrm{~d} s}=-Y^{i} \sum_{j} V_{\alpha}^{j} \frac{\mathrm{d} Y^{j}}{\mathrm{~d} s}
$$

which then define $V_{\alpha}(s)$ via (3) at all $s$. These vectors generate the surface element field, and can now be used to define pseudo-Fermi coordinates $\psi^{i}$ in $U_{\gamma \gamma^{\prime}}$ : simply set

$$
\begin{aligned}
\psi^{n}(x) & =s_{x}, \\
\psi^{\alpha}(x) & =\sum_{j} Z_{x}^{j}{ }_{\alpha} V^{j} .
\end{aligned}
$$

The definition of normal coordinates based on a point $X$ in $G(M)$, the set of all (not necessarily orthonormal) frames, is the usual one [5] with $\partial /\left.\partial x^{i}\right|_{0}=X$.

Suppose $g \in \mathscr{G}^{k}(M)$. For any $\boldsymbol{X} \in L$ and any ordered finite set $\mu$ of integers $\left(j_{1}, j_{2}, \ldots, j_{p}\right)$ with $p \leqq k+2,1 \leqq j_{r} \leqq n$, define

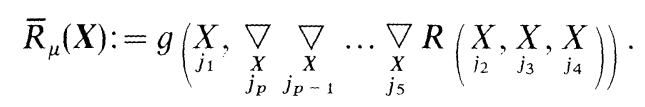

Then if $\kappa: I \rightarrow M$ is a $C^{1}$ immersion with horizontal lift $\gamma$ we define $\bar{R}_{\mu}^{\gamma}(s):=\bar{R}_{\mu}(\gamma(s))$. In this context we shall write $|\mu|$ for $p$, the number of indices, and denote by $P_{n}$ the set of all such $\mu$. For $\mu=\left(j_{1}, \ldots, j_{p}\right)$ we let $i j \ldots k \mu l \ldots q$ denote the set $\left(i, j, \ldots, k, j_{1}, \ldots, j_{p}, l, \ldots, q\right)$.

\section{The Existence of Extensions}

Unless otherwise stated, the following assumption will hold throughout this section:

A. $M$ is an n-manifold, $n \geqq 3, g \in \mathscr{G}^{k}(M)$ for $3 \leqq k \leqq \infty . \kappa: I \rightarrow M$ is a $C^{1}$ (proper $^{1}$ ) embedding parameterised by a generalised affine parameter [4], i.e. there exists a horizontal lift $\gamma$ such that $\sum_{i}\left(Y^{i}\right)^{2}=1\left[Y^{i}\right.$ from (2) and (3)]. $\kappa$ is inextensible at 0 as a $C^{0}$ curve, and so defines a point $q_{\kappa}$ on the b-boundary of $(M, g)$.

We shall say that $M$ is locally $C^{l}$ extensible from $\kappa$ through $q_{\kappa}$ if there exists an open set $U \subset M$ with $\kappa(I) \subset U$, a manifold $M^{\prime}$ with a $g^{\prime} \in \mathscr{G}^{l}\left(M^{\prime}\right)$,

\footnotetext{
${ }^{1}$ In the sense of Nash [6]. In modern terminology, simply an embedding.
} 
and an isometric diffeomorphism $\varphi: U \rightarrow M^{\prime}$ such that $\varphi \kappa$ is extensible at 0 in $M^{\prime}$ as a $C^{0}$ curve.

Suppose this to be the case. Then $\kappa^{\prime}:=\varphi \kappa$ has the horizontal lift $\gamma^{\prime}:=\varphi_{*} \gamma\left(\varphi_{*}: L \mid U \rightarrow L^{\prime}\right.$ is the usual induced map) and $\gamma^{\prime}$ is also extensible at 0 . Hence the numbers $\bar{R}_{\mu}^{\prime \gamma^{\prime}}(s)$ tend to limits as $s \rightarrow 0$. But by the isometry $\bar{R}_{\mu}^{\prime \gamma^{\prime}}=\bar{R}_{\mu}^{\gamma}$, and so a necessary condition for extensibility is the following statement:

B. For every $\mu \in P_{n}$ with $|\mu| \leqq k+2$, the function $\bar{R}_{\mu}^{\gamma}$ tends to a limit $\stackrel{\circ}{R}_{\mu}^{\gamma}$ as s tends to 0 .

We note that for $|\mu| \leqq k+1$

$$
\frac{\mathrm{d}}{\mathrm{d} s} \bar{R}_{\mu}^{\gamma}=Y^{j} \bar{R}_{\mu j}^{\gamma}=Y^{j}\left(\stackrel{\circ}{R}_{\mu j}^{\gamma}+o(1)\right)
$$

where the " $O$ " notation refers to the behaviour as a function of $s$. So

$$
\bar{R}_{\mu}^{\gamma}(s)=\stackrel{\circ}{R}_{\mu}^{\gamma}+\stackrel{\circ}{R}_{\mu j}^{\gamma} z^{j}+\|z\| o(1)
$$

where $z^{j}=\int_{0}^{s} Y^{j} \mathrm{~d} s^{\prime}$ and $\|z\|^{2}=\sum_{i}\left(z^{i}\right)^{2}$. Then, by induction,

$$
\begin{aligned}
\bar{R}_{j_{1} \ldots j_{4}}^{\gamma}= & {\stackrel{\circ}{j_{1} \ldots j_{4}}}^{\gamma}+{\stackrel{\circ}{j_{1} \ldots j_{5}} z^{j_{5}}+\cdots}^{j_{5}}+\frac{1}{(k-2) !}{\stackrel{\circ}{j_{1} \ldots j_{k+2}}}^{\gamma} z^{j_{5}} \ldots z^{j_{k+2}}+\|z\|^{k-2} o(1) .
\end{aligned}
$$

Note that the $z^{i}$ are the coordinates induced on $\kappa(I)$ by an equivalent embedding in the standard flat space of this signature (e.g. Minkowsky space).

As is suggested by (5), and as will be seen later on, the differentiability of a possible extension is determined by the behaviour of the $o$ (1) term, and this in turn depends on the behaviour of $\|z\|$ as a function of $s$. To formulate this we define a spiral curve as an immersion $\kappa: I \rightarrow M$ such that for some (and so for any) horizontal lift $\gamma$ we have $\|z\|=o(s)$. Otherwise the curve is said to be non-spiral. We can now give the main result as follows:

Theorem. Suppose that $M, g, \kappa$ and $\gamma$ satisfy $A$ and $B$ above. Then $M$ is locally $C^{2}$ extensible from $\kappa$ through $q_{\kappa}$. If, in addition, $\kappa$ is a non-spiral curve, then $M$ is locally $C^{k}$ extensible from $\kappa$ through $q_{\kappa}$.

The first stages of the proof can be outlined in the form of three lemmas, whose proofs will be indicated briefly in the last section.

Lemma 1. For any set of numbers $\stackrel{R}{\mu}_{\mu}^{\gamma}$, where $\mu$ runs over $P_{n}$ with $4 \leqq|\mu|<k+3$, satisfying the Bianchi identities

$$
\stackrel{\circ}{R}_{i j k l m v}^{\gamma}+\stackrel{\circ}{R}_{i j l m k v}^{\gamma}+\stackrel{\circ}{R}_{i j m k l v}^{\gamma}=0
$$


for all $v \in P_{n},|v|<k-2$, there is a manifold $M^{\prime}$ and a metric $g^{\prime} \in \mathscr{G}^{\infty}\left(M^{\prime}\right)$ having these numbers as the components of the covariant derivatives of the Riemann tensor with respect to some $\mathrm{X} \in L^{\prime}$.

Lemma 2. Let $\kappa$ be as in $A$. For any manifold $M^{\prime}$, any $g^{\prime} \in \mathscr{G}^{l}\left(M^{\prime}\right), l \geqq 2$, and any point $\boldsymbol{X} \in L^{\prime}$ there is a horizontal curve $\gamma^{\prime}:\left(0, s_{0}\right) \rightarrow L^{\prime}$ which is equivalent to $\gamma \mid\left(0, s_{0}\right)$ and has $\operatorname{Lim}_{s \rightarrow 0} \gamma^{\prime}(s)=\boldsymbol{X}$.

We note that we can always rescale the generalised affine parameter by choosing a different lift, altering $\boldsymbol{X}$ by a pseudo-orthogonal transformation (e.g. Lorentz). Thus without loss of generality we can take $s_{0}=1$.

Lemma 3. Let $\kappa^{\prime}$ be a $C^{1}$ immersion of $(0,1)$ in $M^{\prime}$ having a horizontal lift $\gamma^{\prime}$ for $g^{\prime} \in \mathscr{G}^{l}\left(M^{\prime}\right)$ such that $\operatorname{Lim}_{s \rightarrow 0} \gamma^{\prime}(s)$ exists. Then there is a metric $g^{\prime \prime} \in \mathscr{G}^{\prime}\left(M^{\prime}\right)$ and a horizontal curve $\gamma^{\prime \prime}: I \rightarrow L^{\prime \prime}$ such that:

(i) $\gamma^{\prime \prime}$ is equivalent to $\gamma$

(ii) $\pi^{\prime \prime} \gamma^{\prime \prime}$ is a (proper) embedding, where $\pi^{\prime \prime}: L^{\prime \prime} \rightarrow M^{\prime}$ is the bundle projection

(iii) $\operatorname{Lim} i^{\prime \prime} \gamma^{\prime \prime}(s)=\operatorname{Lim} i^{\prime} \gamma^{\prime}(s)$ where $i^{\prime}$ (resp. $\left.i^{\prime \prime}\right)$ is the inclusion of $L^{\prime}$ (resp. $L^{\prime \prime}$ ) in $G(M)$

(iv) $g^{\prime}-g^{\prime \prime}$ makes $m^{\prime}$ th order contact with the zero cross section at $\pi^{\prime} X$ (i.e. all derivatives exist and are zero).

We thus acheive a horizontal curve $\gamma^{\prime \prime}$ equivalent to $\gamma$, whose projection is a proper embedding and which satisfies $\bar{R}_{\mu}^{\prime \prime \gamma^{\prime \prime}} \rightarrow R_{\mu}^{\gamma}$ for all $\mu \in P_{n}$, $4 \leqq|\mu|<k+3$. Now we alter the metric in the set $V^{\prime \prime}:=U_{\gamma \gamma^{\prime \prime}}^{\prime}$ so that $\bar{R}_{\mu}^{\gamma^{\prime \prime}}$ becomes equal to $\bar{R}_{\mu}^{\gamma}$, while leaving $\gamma^{\prime \prime}$ equivalent to $\gamma$. To see that this is possible, take pseudo-Fermi coordinates in $V^{\prime \prime}$ and require that the metric have the same first derivatives as the old metric on $\kappa(I)$. Then the derivatives $\left.g_{i j, v}\right|_{\kappa(I)}$ for $v \in P_{n}, 2 \leqq|v|<k+1$ are uniquely specified by an algebraic relation in terms of the Riemann tensor and its covariant derivatives.

It thus follows that we can choose this alteration $\delta g$ to vanish outside a tube $\left\|\psi^{\alpha}\right\|<\varepsilon\left(\psi^{n}\right)$, and we can then select $\varepsilon$ small enough to give $\delta g_{i j, k}<\|x\|^{2}$ in normal coordinates based on $\boldsymbol{X}$. This means that the new metric will be $C^{2}$, since all the directional derivatives of $\delta g_{i j, k}$ vanish at the point $O:=\pi^{\prime} X$ and tend to zero, as this point is approached.

If, however, the curve is not spiral then the " $O$ " term in Eq. (5) becomes $o\left(\|z\|^{k-2}\right)$. Moreover, it is simple to prove the following:

Lemma 4. If $\kappa^{\prime}: I \rightarrow M^{\prime}$ is a non-spiral curve (immersion) for $g^{\prime} \in \mathscr{G}^{k}\left(M^{\prime}\right)$ and $\left\{x^{i}\right\}$ are $C^{k}$ coordinates about $\operatorname{Lim}_{s \rightarrow 0} \kappa^{\prime}(s)$, then $\exists K^{\prime}, s_{1}$ such that for $s<s_{1},\|x\|>K^{\prime}\|z\|$. 
Thus the " $o$ " term becomes $o\left(\|x\|^{k-2}\right)$ with $\|x\|$ of the same order as $s\left(K^{\prime \prime} s<\|x\|<K^{\prime \prime \prime} s\right)$. It follows immediately that the altered metric is now $C^{k}$.

The final step is to map a subset of $U_{\gamma \gamma^{\prime}}$ into $V^{\prime \prime}$ by the map $\tau$, thereby establishing a metric $g^{*}:=\left(\tau^{*}\right)^{-1} g$ in the tube $\left\|\psi^{\alpha}\right\|<\varepsilon\left(\psi^{n}\right) / 2$. This metric can be joined smoothly onto $g^{\prime \prime}$ outside $\left\|\psi^{\alpha}\right\|<\varepsilon\left(\psi^{n}\right)$. We must now show that the resulting metric, call it $\hat{g}$, is in general $C^{2}$, and for non-spiral curves $C^{k}$.

Choose normal coordinates $x^{j}$ relative to $X$ and consider a point $P^{\prime}=\left\{x^{i}\right\}=\kappa^{\prime}\left(s_{1}\right)$. Then set up normal coordinates $\left\{w^{i \prime \prime}\right\}$ and $\left\{w^{i *}\right\}$ both based on $\boldsymbol{X}\left(s_{1}\right)$ but using the metrics $g^{\prime \prime}$ and $g^{*}$ respectively. Then for the coordinates of a point $Q$ sufficiently near $P^{\prime}$ we have

$$
\left\|w^{\prime \prime}-w^{*}\right\|<H\|z\|^{k}
$$

where $H$ depends on the Riemann tensor and its derivatives, i.e. on the $\bar{R}_{\mu}^{\gamma}$ at $s_{1}$. But the components of the metrics in their proper normal coordinates are given by series expansions with coefficients depending on the $\bar{R}_{\mu}^{\gamma}\left(s_{1}\right)$ which are the same for both metrics up to order $k$. Thus (6) implies that the coordinates of the two metrics in a single normal coordinate system, e.g. $w^{\prime \prime}$, are related by

$$
\left\|g_{i j}^{*}-g_{i j}^{\prime \prime}\right\|<M\left(\bar{R}_{\mu}^{\gamma}\left(s_{1}\right)\right)\|z\|^{k} .
$$

Similarly, we have for the derivatives

$$
\left\|\partial_{\mu} g_{i j}^{*}-\partial_{\mu} g_{i j}^{\prime \prime}\right\|<N\|z\|^{k-r}, \quad \text { for } r=|\mu|<k,
$$

where $N$ can be taken absolutely constant.

Now we transform back to the normal coordinates $x^{i}$. The transformation depends only on the two basis frames $\boldsymbol{X}$ and $\boldsymbol{X}\left(s_{1}\right)$, not on any other property of $\gamma$, and so it follows that (7) holds with the left hand side evaluated in the coordinates $x^{i}$. Differentiability now follows on choosing a smaller tubular neighbourhood, if necessary, and using the same argument as for the previous alteration in the metric.

Corollary. If $M, g, \kappa$ and $\gamma$ satisfy $A$. and $B$., with $(M, g)$ being a spacetime and $\kappa$ a causal curve, then $M$ is locally $C^{k}$ extensible from $\kappa$ through $q_{\kappa}$.

Proof. The embedding $\kappa^{\prime}$ in Minkowsky space equivalent to $\kappa$ is also a causal curve, and so satisfies $\|z\| \geqq s / \sqrt{2}$. It follows that $\kappa$ is not spiral.

\section{A Spiral Curve without a $C^{k}$ Extension}

We complete the discussion by demonstrating the necessity of the restriction to non-spiral curves by an example of a curve which satisfies 
A. and B. but whose boundary point $q_{\kappa}$ does not admit a $C^{l}$ extension from $\kappa$ for any $l>2$.

First take the positive definite metric on $R^{2} d s^{2}=(1+x)^{2}\left(d x^{2}+d y^{2}\right)$, having $\partial / \partial y$ as a Killing vector and non-zero Riemann tensor and first derivative at the origin $\left(R_{1212}=-1, R_{1212 ; 1}=4\right)$. Take for $\kappa_{0}$ a curve in $R^{2}$ which keeps returning to the origin, such as that obtained by describing successively the sides of a sequence of squares in the positive quadrant with two sides on the axes, the $n$ 'th square having sides of length $1 / 2^{n}$. Define $\gamma_{0}$ to be that horizontal lift of $\kappa$ whose 0 -endpoint is $(\partial / \partial x, \partial / \partial y)$ and let $s$ denote the generalised affine parameter for this lift. As usual $Y^{i}(s)=\theta^{i}\left(\dot{\gamma}_{0}\right)$.

Next construct a flat 2 -space $K$ containing a properly embedded curve $\kappa$ equivalent to $\kappa_{0}$. This can be done by first taking an immersion $\kappa_{1}$ in Euclidean space $E^{2}$ equivalent to $\kappa_{0}$, and then mapping $R^{2}$ to $E^{2}$ diffeomorphically by a map $\varphi$ such that for all $s \in I$ there is a unique $x_{s} \in R$ with $\kappa_{1}(s)=\varphi\left(x_{s}, s\right)$. The required space $K$ is the inverse image of the metric space $E^{2}$ under $\varphi$ (i.e. $R^{2}$ with metric induced by $\varphi^{*}$ ) and $\kappa(s)=\left(x_{s}, s\right)$.

If we now alter the metric on $K$ while keeping it and its first derivatives on $\kappa$ unchanged then, as shown in the previous section, we can achieve a Riemann tensor which gives the following behaviour for $\bar{R}_{\mu}^{\gamma}$ :

$$
\begin{aligned}
& \bar{R}_{1212}^{\gamma} \rightarrow-1 \quad \bar{R}_{12122}^{\gamma}=0 \\
& \bar{R}_{12121}^{\gamma}=4
\end{aligned}
$$

while leaving $\kappa$ equivalent to $\kappa_{0}$. Since the Bianchi identities are trivially satisfied as a consequence of the Riemann tensor symmetries there are no constraints on the Riemann tensor and any number of other derivatives transverse to the curve may be specified arbitrarily.

To form a space-time we now take the metric product of $K$ with two-dimensional Minkowsky space. The dimension of the manifold will not enter the discussion here (although it played a role in the proof of the theorem) and so to simplify the formulation we give a description only in terms of the two-space $K$.

Suppose that this space-time had a local extension from $\kappa$ through $q_{\kappa}$. Then in the extension we can take normal coordinates $x^{\prime i}$ about the endpoint so that they differ on the curve from the original coordinates $x^{i}=(x, y)$ according to

for some $N_{1}$.

$$
\left|x^{i}-x^{\prime}\right|<N_{1} s^{4}
$$

But the Riemann tensor on the curve is governed by

$$
\bar{R}_{1212}=\int Y^{1} \bar{R}_{12121} d s=4 z^{1}
$$


and it is easily calculated that, for some $N_{2},\left|z^{1}-x^{1}\right|>N_{2} s^{3}$. Thus from (9) we see that at points where $x^{i}=0$

$$
\left|\stackrel{\circ}{R}_{1212}-\bar{R}_{1212}\right|>\left(4 N_{2} / N_{1}\right)\left\|x^{\prime}\right\|^{4 / 3} .
$$

But this occurs for arbitrarily small values of $s$, whenever the original curve returns to the origin. Thus the Riemann tensor cannot be differentiable at the origin in the extension, because it is given from the $\bar{R}_{\mu}^{\gamma}$ through the tetrad $\boldsymbol{X}$ whose coordinate components satisfy $\underset{i}{X_{j}^{j}}=\delta_{i}^{j}+O\left(s^{2}\right)$. Thus the extension is at most $C^{2}$.

We note that the situation has a certain stability: sufficiently small variations, both of the curve and of the metric, do not affect the nonextensibility.

\section{Proofs of Lemmas}

Proof of Lemma 1. Since the derivatives at the origin of the components of the metric in normal coordinates are determined by the covariant derivatives of the Riemann tensor at the origin, and vice versa, the problem reduces to that of finding a function $g\left(=g_{i j}\right)$ on $R^{n}$ with specified derivatives $g_{\mu}^{0}$ at the origin. If the Taylor series converges in a neighbourhood of the origin there is no problem; if it does not converge take

$$
g=\sum_{p} \sum_{|\mu|=p}\left(1 /\left(q_{\mu}\right)^{p}\right) \prod_{i=1}^{p} f\left(q_{\mu} g_{\mu}^{01 / p} x^{k_{k}}\right)
$$

where $\mu=\left(k_{1}, \ldots, k_{p}\right)$

$$
q_{\mu}=\operatorname{Max}\left[g_{\mu}^{0}, 1\right]\left(2 n p \operatorname{Max}_{j<p} \operatorname{Sup}_{x} f^{(j)}(x)\right)^{p}
$$

and $f$ is a $C^{\infty}$ function $R \rightarrow R$ satisfying

$$
f(x)= \begin{cases}x & |x|<1 / 2 \\ x /|x| & |x|>3 / 2 .\end{cases}
$$

Each term in the series becomes equal to the corresponding term in a Taylor expansion for small enough $x$, while the choice of $f$ and the $q_{\mu}$ ensures that any derivative of the series will converge uniformly.

Proof of Lemma 2. $\gamma^{\prime}$ must satisfy the ordinary differential equation

$$
\dot{\gamma}^{\prime}=\sum_{i} Y^{i}(s) \tilde{X}_{i}^{\prime}(s)=: f_{Y}\left(\gamma^{\prime}(s), s\right)
$$

where $\tilde{X}_{i}^{\prime}$ are the standard horizontal vector fields dual to the $\theta^{i}$. We cannot immediately take $\boldsymbol{X}$ as the initial value for this equation, since the $Y^{i}$ are not continuous at $s=0$. Instead, we must take an initial value $\boldsymbol{X}_{0}$ and integrate the equation in the direction of decreasing $s$, choosing 
$\boldsymbol{X}_{0}$ so as to arrive at $\boldsymbol{X}$ when $s=0$. The existence of such an $\boldsymbol{X}_{0}$ follows from a contraction-mapping argument.

First we restrict attention to a neighbourhood $U$ of $\boldsymbol{X}$ in which for any functions $Y^{i}$

$$
\left\|f_{Y}(y, s)\right\| \leqq P \quad \forall s \in I, \forall y \in U
$$

(where the norm is evaluated in the bundle metric, or in a coordinate system). Then we restrict the range of $s$ and the distance of $\boldsymbol{X}_{0}$ from $\boldsymbol{X}$ so that the development of (10) remains in $U$. Now, measuring norms in some coordinate system $\left\{y^{\beta}\right\}$ in the frame bundle, there will be a constant $K$ such that for $\boldsymbol{W}_{1}, \boldsymbol{W}_{2}$ in $U$

$$
\left\|\tilde{X}_{i}\left(\boldsymbol{W}_{1}\right)^{\beta}-\tilde{X}_{i}\left(\boldsymbol{W}_{2}\right)^{\beta}\right\|<K\left\|W_{1}^{\beta}-W_{2}^{\beta}\right\| .
$$

Let $\gamma^{\prime}, \gamma^{\prime \prime}$ be two solutions of (10). Then for small enough $s_{0}$, depending only on $U$,

$$
s_{1}, s_{2}<s_{0} \Rightarrow\left\|\gamma^{\prime}\left(s_{1}\right)^{\beta}-\gamma^{\prime \prime}\left(s_{1}\right)^{\beta}\right\| \leqq e^{K\left|s_{1}-s_{2}\right|}\left\|\gamma^{\prime}\left(s_{2}\right)^{\beta}-\gamma^{\prime \prime}\left(s_{2}\right)^{\beta}\right\| .
$$

We can take $s_{0}<(1 / K) \cdot \log (3 / 2)$. Then the mapping $\alpha$ defined by

$$
\alpha\left(\boldsymbol{X}_{0}\right)^{\beta}=\boldsymbol{X}_{0}^{\beta}+\boldsymbol{X}^{\beta}-\gamma^{\prime}(0)^{\beta}
$$

[where $\beta$ denotes components relative to the chosen coordinate system and $\left.\gamma^{\prime}(0)=\operatorname{Lim}_{s \rightarrow 0} \gamma^{\prime}(s)\right]$ is a contraction mapping and defines the required $\boldsymbol{X}_{0}$ by iteration.

The same arguments easily give the following

Lemma 5. Let $g_{i j}, g^{\prime}{ }_{i j}$ be the components of two metrics on an open set $U \subset M$ with respect to some fixed coordinate system, and let $\gamma, \gamma^{\prime}$ be equivalent horizontal immersions in the frame bundles with the same endpoint in $G(M)$. Then there are constants $K, s_{0}, P$ depending only on $g$ such that for $0<s<s_{0}$ and

we have

$$
\sum_{i j, k}\left|g_{i j, k}^{\prime}-g_{i j, k}\right|^{2}=: \varphi^{2}<P
$$

$$
\left\|\gamma^{\beta}-\gamma^{\prime \beta}\right\|<K \varphi s .
$$

We note that Lemma 4 follows from this.

Proof of Lemma 3. Choose normal coordinates for $\left(M^{\prime}, g^{\prime}\right)$ relative to $X$ and in a closed ball $V$ in these coordinates consider the set of metrics of the form

$$
g_{i j}^{\prime \prime}=g_{i j}^{\prime}+h_{i j}
$$

where the $h_{i j}$ are $C^{\infty}$ functions having all their derivatives zero at the origin. The set of all such $h_{i j}$ forms a complete metric space (in fact a Frechet topological vector space) in which the $h_{i j}$ such that $g^{\prime \prime}$ is a metric of the required signature form an open set $\mathbf{6}$ which we identify with the 
corresponding set of metrics $g^{\prime \prime}$. Moreover, we can find a neighbourhood $\mathfrak{H}$ of $g^{\prime}$ whose closure lies in $\mathfrak{6}$. It suffices to show that the set of elements of $\mathbf{6}$ for which the equivalent curve $\gamma^{\prime \prime}$ is a (proper) embedding is dense in the complete metric space $\overline{\mathfrak{H}}$.

Take a closed interval $J=[a, b] \subset I$ and consider $\gamma_{J}:=\gamma^{\prime \prime} \mid J$. Then we show that the subset $\mathfrak{G}_{J}$ of $\mathfrak{b}$ for which $\kappa_{J}:=\pi \gamma_{J}$ is not self-intersecting and does not pass through the origin is an open dense set. This is clearly the case for the set of metrics for which there is no passage through the origin, and so we need only consider the self-intersections. From Lemma 5 the quantity

$$
\operatorname{Inf}_{s_{1}, s_{2} \in J} \frac{\left\|\kappa^{i}\left(s_{1}\right)-\kappa^{i}\left(s_{2}\right)\right\|}{\left|s_{1}-s_{2}\right|}
$$

depends continuously on the metric $g^{\prime \prime}$ and is non-zero when $\pi \gamma_{J}$ is not self intersecting. Thus $\mathfrak{6}_{J}$ is open. Suppose that it is not dense in $\overline{\mathfrak{H}}$, i.e. that there is an open set $\mathfrak{B}$ in $\mathfrak{b}$ in which all metrics give rise to intersections. Let $g_{0}$ be a point in this set and $\kappa_{0}$ the corresponding curve. The continuity of $\kappa_{0}$ in the compact interval $J$ ensures that there is an element $\zeta$ of $R^{n}$ with $\sum_{\alpha} \zeta^{\alpha} \zeta^{\alpha}=1$ such that all intersections are removed by all sufficiently small displacements of small parts of the curve in the direction $\zeta$, or sufficiently near $\zeta$. Then all metrics of the form

$$
g_{i j}^{\prime \prime}=g_{i j}^{\prime}+\varepsilon f\left(\sum_{\alpha} \zeta^{\alpha} z^{\alpha}\right)\left(\delta_{i j}-\zeta^{i} \zeta^{j}\right)
$$

for a strictly monotonic $f$ and small enough $\varepsilon$ will be free from intersection.

Thus $\mathfrak{6}_{J}$ must be an open dense set. To complete the proof, we now need only take a countable sequence $\left\{J_{i}\right\}$ of intervals covering $I$. From Baire's theorem $\bigcap_{i}\left(\mathfrak{G}_{J_{\mathfrak{\imath}}} \cap \overline{\mathfrak{S}}\right)$ is dense in $\mathfrak{H}$ and the lemma is proved.

\section{References}

1. Geroch, R.: Ann. Phys. 48, 526 (1968)

2. Schmidt, B. G.: General Relativity and Gravitation, 1, 269 (1971)

3. Ellis, G. F. R., Schmidt, B. G.: To appear

4. Ellis, G.F.R., Hawking, S. W.: The Large Scale Structure of Space-Time, Cambridge University Press 1973

5. Schouten, T.A.: Ricci Calculus, Berlin-Göttingen-Heidelberg: Springer 1954

6. Nash, J.: Ann. Math. 60, 383 (1954)

C. J. S. Clarke

Department of Applied Mathematics and Theoretical Physics

Cambridge, U. K. 